\title{
Spondylodiscitis and an aortic aneurysm due to Campylobacter coli
}

\author{
Xavier Lemaire ${ }^{*}$, Caroline Dehecq ${ }^{1}$, Christian Cattoen ${ }^{2}$, Laurence Destrieux Garnier ${ }^{3}$, Béatrice Sarraz Bournet ${ }^{4}$, \\ Yazdan Yazdanpanah", Eric Senneville ${ }^{1}$
}

\section{Abstract}

Campylobacter coli is a rare cause of bacteremia. We report here the first case of C.coli spondylodiscitis complicated by an aortic aneurysm. Outcome was favourable with surgery and antibiotic therapy.

\section{Background}

While Campylobacter fetus is a rare but well-recognized cause of bacteremia [1-3], only a few cases of Campylobacter coli bacteremia have been reported in the literature. To the best of our knowledge, no aortic aneurysm due to C.coli has been previously described. We report here the first case of C.coli spondylodiscitis complicated by an aortic aneurysm.

\section{Case Presentation}

A 72-year old man was admitted in June 2006 in the Rheumatology Department of the General Hospital of Valenciennes-France, for a history of lumbar pain evolving for one month. He had a previous medical history of myocardial injury, dyslipidaemia and tobacco intoxication; he had neither diabetes mellitus nor immunosuppressive therapy. The patient lived in a city and had only a dog. No chills or rigors, but several episodes of nocturnal fever, were noted. At admission, the patient was afebrile, and reported only lumbar back pain and sciatic pain. White blood cell count was 13,000 G/L, ESR $89 \mathrm{~mm} / \mathrm{h}$ and CRP $138.5 \mathrm{mg} / \mathrm{L}$. Blood cultures taken at admission grew a Gram-negative bacillus. The strain was identified as C.coli with the API Campy identification system, and was susceptible to all tested antibiotics. Treatment with a combination of cefotaxime plus gentamicin was immediately started. MRI showed abnormalities consistent with a L4-L5 spondylodiscitis associated with prevertebral and left psoas abscesses. Abdominal CT scan showed a ruptured abdominal aortic aneurysm and a contiguous left psoas collection

\footnotetext{
* Correspondence: xljhe@yahoo.fr

'Department of Infectious Diseases, CH Dron Tourcoing, France
}

(figure 1,2). The patient was transferred to the University Hospital of Lille to be operated on for insertion of endovascular prosthesis. He was then transferred to our Infectious Disease Unit where antibiotic treatment for the aneurysm and spondylodiscitis was continued.

In our unit, the patient remained afebrile without pain. Nevertheless, C-reactive protein continued to markedly rise to $105 \mathrm{mg} / \mathrm{L}$. Ciprofloxacin was added to cefotaxime, and gentamicin was stopped after two weeks of treatment. The day after his admission to our unit, septic shock occurred. CT scan showed the persistence of a $10 \times 7.7 \times 3.7 \mathrm{~cm}$ peri-aortical collection, maybe due to a hematoma related to the first surgical intervention. Surgical drainage was immediately performed (cultures from intraoperative samples were sterile). Postoperative evolution was favourable except for slight resolvable renal failure. CT scan carried out two weeks after surgery showed a marked reduction in the size of the collection and a decrease in the CRP level to $16 \mathrm{mg} /$ $\mathrm{L}$ at the same time. Transoesophagal echocardiography was normal. A coloscopy was performed which revealed only a $5 \mathrm{~mm}$ non-resecable caecal polyp. Cefotaxime was stopped after two weeks and ciprofloxacin was switched to oral form and is still ongoing. 4 months after surgery, the CT scan showed the disappearance of the initial collection. Two years later, the patient continues to be in good health and remains totally asymptomatic.

\section{Discussion}

Except for one study [4], the incidence of C.coli bacteremia remains unknown [5] but seems to be very rare. This is likely due to the susceptibility of this pathogen to the antibacterial activity of the serum [6]. This could 


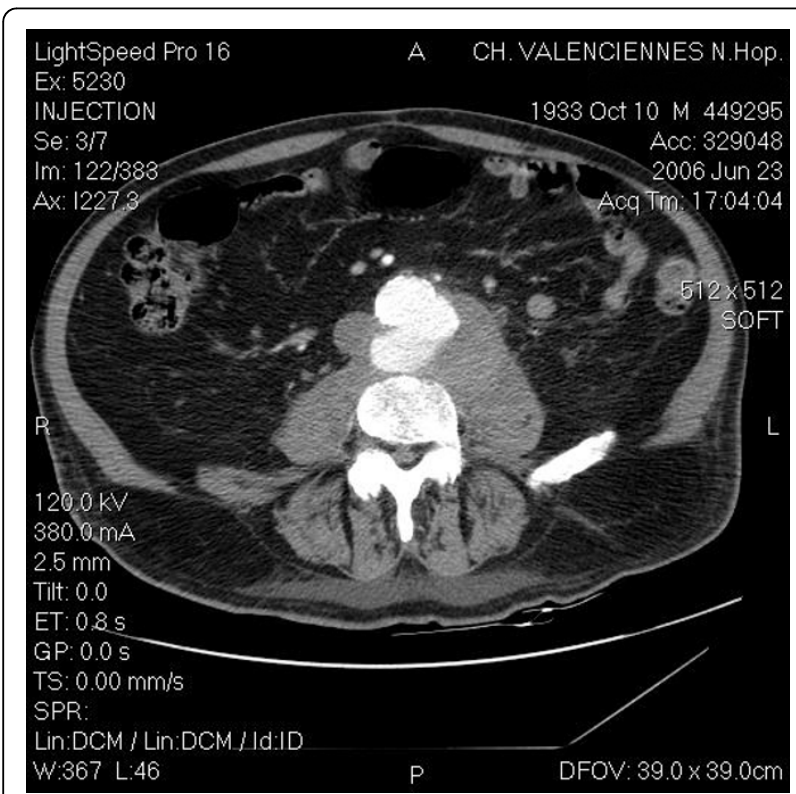

Figure $1 \mathrm{An}$ axial CT image (the first to be performed for this patient, at the General Hospital, Valenciennes-France) with contrast of the lumbar area showing abdominal aortic aneurysm.

explain why C.coli bacteremia episodes are often reported in immunocompromised patients or in children [5,7-12]. Only a few studies report bacteremia occurring in immunocompetent patients during acute enteritis $[4,13]$. Therefore, the incidence of C.coli bacteremia may be underestimated due to infrequent blood sampling at the early stage of infection, and inappropriate culture conditions [14].

Although bacteremia may be misdiagnosed, aortic aneurysm and spondylodiscitis are more highly visible infections. Nevertheless, no cases of these localizations have been reported for C.coli. We found only one case of meningitis due to this pathogen, but observed no arthritis, endocarditis, pneumonia or prostatitis. We have no evidence for an associated endocarditis and colonoscopy revealed only a small non-inflammatory polyp. We also did not find any cause of immunosuppression in our patient except for age $>65$ years. Skirrow et al [4] found the average incidence of Campylobacter bacteremia to be $1.5 / 1,000$ intestinal Campylobacter infections in their total population, and $5.9 / 1000$ in patients aged 65 or over.A combination of cephalosporin and gentamicin was chosen in an attempt to obtain a synergistic bactericidal effect during the first ten days of treatment. Ciprofloxacin was prescribed as prolonged antibiotic therapy in an attempt to obtain permanent high concentrations at the infected site. Although a 3 months duration of treatment for abdominal aortic aneurysms infected with Campylobacter has been evocated in two cases $[15,16]$, considering that an endovascular prosthesis had been inserted in an infected site and the risk of relapsing infection, we considered a life-long antibiotic suppressive therapy as reported for other bacteria $[17,18]$. A prolonged course of ciprofloxacin was then proposed to the patient due to its activity toward foreign body infection due to Gram-negative bacilli, as demonstrated in both experimental and clinical studies [19-22].

Concerning the spondylodiscitis, despite some reported studies [23-25], as drainage of the prevertebral and left psoas abscesses was done during the first surgical intervention; no specific intervention was then performed.

We believe that the spondylodiscitis was primarily due to C.coli bacteremia, but it is unclear as to whether the aneurysm rupture was secondary to bacteremia or to erosion of the arterial wall by the abscess.

A limitation of our case report is that species identification was made with the API Campy identification system and not by PCR. As previously reported the sensibility of the API Campy test, particularly for identification of C.coli, is poor [26-28]. Despite imperfect specificity of this test, diagnosis of C.coli was always confirmed by PCR in these studies. In accordance with these data, the possibility of misdiagnosing Campylobacter species in the present case seems to be very low.

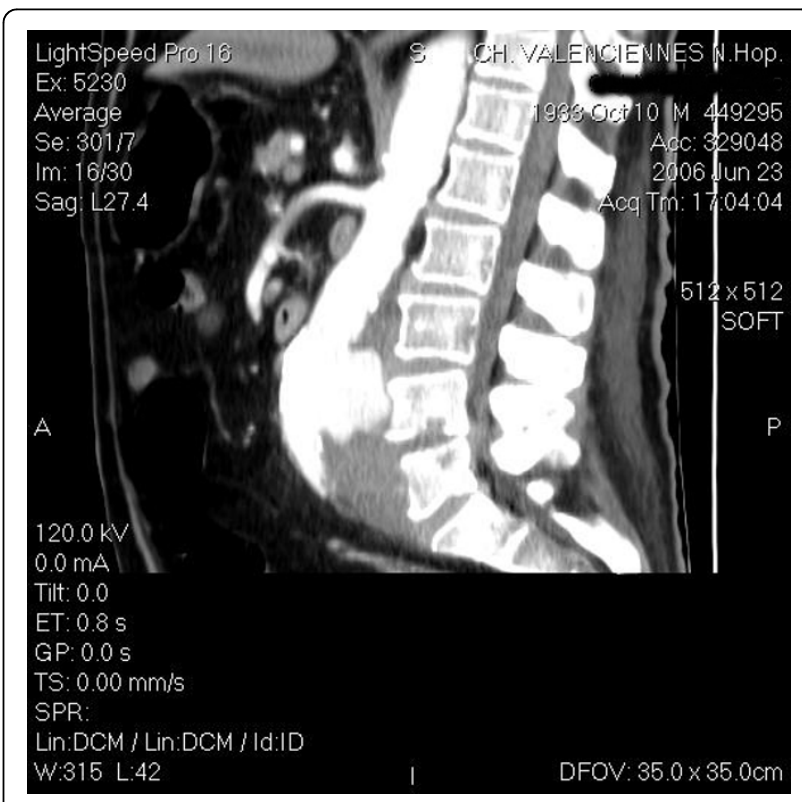

Figure $2 \mathrm{~A}$ sagittal CT image of the lumbar area with contrast reconstruction showing abdominal aortic aneurysm. 


\section{Conclusion}

Although it had not yet been described, C.coli may be responsible for spondylodiscitis complicating bacteremia. Like C.fetus, C.coli may, under conditions not yet elucidated, cross the intestinal barrier and cause severe metastatic infections.

\section{Consent}

Written informed consent was obtained from the patient for publication of this case report and any accompanying images. A copy of the written consent is available for review by the Editor-in-Chief of this journal.

\section{Author details}

${ }^{1}$ Department of Infectious Diseases, CH Dron Tourcoing, France.

2Department of Microbiology, CH Valenciennes, Valenciennes, France. ${ }^{3}$ Department of Cardio-Vascular Surgery, Hopital cardiologique, Lille, France.

${ }^{4}$ Department of Cardio-Vascular Surgery, CH Dron, Tourcoing, France.

\section{Authors' contributions}

$\mathrm{XL}$ have made substantial contributions to acquisition of data, have been involved in drafting the manuscript. CD have been involved in drafting the manuscript. CC have made substantial contributions to acquisition of data. LDG have made substantial contributions to acquisition of data. BSB have made substantial contributions to acquisition of data. $Y Y$ have given final approval of the version to be published. ES have made substantial contributions to conception of the case redaction, have given final approval of the version to be published. All authors read and approved the final manuscript.

\section{Competing interests}

The authors declare that they have no competing interests.

\section{Received: 1 December 2009}

Accepted: 5 February 2010 Published: 5 February 2010

\section{References}

1. Nielsen $H$, Hansen KK, Gradel KO, Kristensen B, Ejlertsen T, Ostergaard C, Schonheyder HC: Bacteraemia as a result of Campylobacter species: a population-based study of epidemiology and clinical risk factors. Clin Microbiol Infect 16(1):57-61.

2. Pacanowski J, Lalande $V$, Lacombe $K$, Boudraa $C$, Lesprit $P$, Legrand $P$, Trystram D, Kassis N, Arlet G, Mainardi JL, et al: Campylobacter bacteremia: clinical features and factors associated with fatal outcome. Clin Infect Dis 2008, 47(6):790-796.

3. Gazaigne L, Legrand P, Renaud B, Bourra B, Taillandier E, Brun-Buisson C, Lesprit P: Campylobacter fetus bloodstream infection: risk factors and clinical features. Eur J Clin Microbiol Infect Dis 2008, 27(3):185-189.

4. Skirrow MB, Jones DM, Sutcliffe E, Benjamin J: Campylobacter bacteraemia in England and Wales, 1981-91. Epidemiol Infect 1993, 110(3):567-573.

5. Ladron de Guevara C, Gonzalez J, Pena P: Bacteraemia caused by Campylobacter spp. J Clin Pathol 1994, 47(2):174-175.

6. Blaser MJ, Smith PF, Kohler PF: Susceptibility of Campylobacter isolates to the bactericidal activity of human serum. J Infect Dis 1985, 151(2):227-235.

7. Brunel V, Allegre T, Cailleres S, Blanc AP: Hematologic manifestations in Campylobacter coli septicemia. Apropos of a case. Rev Med Interne 1993, 14(1):39-40.

8. Schonheyder HC, Sogaard P, Frederiksen W: A survey of Campylobacter bacteremia in three Danish counties, 1989 to 1994 . Scand J Infect Dis 1995, 27(2):145-148.

9. Pigrau C, Bartolome R, Almirante B, Planes AM, Gavalda J, Pahissa A: Bacteremia due to Campylobacter species: clinical findings and antimicrobial susceptibility patterns. Clin Infect Dis 1997, 25(6):1414-1420.

10. Akiba T, Akiba K, Suto N, Kumagai K, Sakamoto M, Yazaki N: Campylobacter coli bacteremia in an 11-year-old boy. Pediatr Int 2002, 44(5):543-544.
11. Le Risbe C, Bosseray A, Leclerq P, Pegourie B, Megraud F, Croize J: Relapsing Campylobacter coli bacteremia in a hypogammaglobulinemic patient. Presse Med 1998, 27(22):1103-1104.

12. Tokuda K, Nishi J, Miyanohara H, Sarantuya J, Iwashita M, Kamenosono A, Hizukuri K, Wakimoto N, Yoshinaga M: Relapsing cellulitis associated with Campylobacter coli bacteremia in an agammaglobulinemic patient. Pediatr Infect Dis J 2004, 23(6):577-579.

13. Vandewal W: Report a case of Campylobacter coli bacteremia in a patient with gastro-enteritis. Acta Clin Belg 2000, 55(6):344.

14. Ketley JM: Pathogenesis of enteric infection by Campylobacter. Microbiology 1997, 143(Pt 1):5-21.

15. Mii S, Tanaka K, Furugaki K, Sakata H, Katoh H, Mori A: Infected abdominal aortic aneurysm caused by Campylobacter fetus subspecies fetus: report of a case. Surgery today 1998, 28(6):661-664.

16. Roan JN, Ko WC, Luo CY: Abdominal septic aortic pseudoaneurysm caused by Campylobacter jejuni infection: report of a case. Surgery today 2009, 39(2):137-140.

17. Berdal JE, Steinbakk M: Long-term antibiotic suppressive therapy for an infected thoracic aorta graft. Tidsskr Nor Laegeforen 2003, 123(16):2260-2262.

18. Terpling S, Schade Larsen C, Schonheyder HC: Long-term home-based parenteral antibiotic treatment of a prosthetic vascular graft infection caused by Pseudomonas aeruginosa. Scand J Infect Dis 2006, 38(5):388-392.

19. Greenberg RN, Newman MT, Shariaty S, Pectol RW: Ciprofloxacin lomefloxacin, or levofloxacin as treatment for chronic osteomyelitis. Antimicrob Agents Chemother 2000, 44(1):164-166.

20. Galanakis N, Giamarellou H, Moussas T, Dounis E: Chronic osteomyelitis caused by multi-resistant Gram-negative bacteria: evaluation of treatment with newer quinolones after prolonged follow-up. J Antimicrob Chemother 1997, 39(2):241-246.

21. Chuard C, Herrmann M, Vaudaux P, Waldvogel FA, Lew DP: Successful therapy of experimental chronic foreign-body infection due to methicillin-resistant Staphylococcus aureus by antimicrobial combinations. Antimicrob Agents Chemother 1991, 35(12):2611-2616.

22. Brouqui P, Rousseau MC, Stein A, Drancourt M, Raoult D: Treatment of Pseudomonas aeruginosa-infected orthopedic prostheses with ceftazidime-ciprofloxacin antibiotic combination. Antimicrob Agents Chemother 1995, 39(11):2423-2425.

23. Woertgen C, Rothoerl RD, Englert C, Neumann C: Pyogenic spinal infections and outcome according to the 36-item short form health survey. Journal of neurosurgery 2006, 4(6):441-446.

24. Nather A, David V, Hee HT, Thambiah J: Pyogenic vertebral osteomyelitis: a review of 14 cases. Journal of orthopaedic surgery (Hong Kong) 2005, 13(3):240-244

25. Turgut M: Complete recovery of acute paraplegia due to pyogenic thoracic spondylodiscitis with an epidural abscess. Acta neurochirurgica 2008, 150(4):381-386.

26. Rautelin $\mathrm{H}$, Jusufovic J, Hanninen ML: Identification of hippurate-negative thermophilic campylobacters. Diagnostic microbiology and infectious disease 1999, 35(1):9-12.

27. Huysmans MB, Turnidge JD, Williams JH: Evaluation of API Campy in comparison with conventional methods for identification of thermophilic campylobacters. Journal of clinical microbiology 1995 33(12):3345-3346.

28. Asakura M, Samosornsuk W, Taguchi M, Kobayashi K, Misawa N, Kusumoto M, Nishimura K, Matsuhisa A, Yamasaki S: Comparative analysis of cytolethal distending toxin (cdt) genes among Campylobacter jejuni, C. coli and C. fetus strains. Microbial pathogenesis 2007, 42(5-6):174-183.

\section{doi:10.1186/1476-0711-9-8}

Cite this article as: Lemaire et al: Spondylodiscitis and an aortic aneurysm due to Campylobacter coli. Annals of Clinical Microbiology and Antimicrobials 2010 9:8. 